\title{
Pupillometry in congenital central hypoventilation syndrome (CCHS): quantitative evidence of autonomic nervous system dysregulation
}

\author{
Pallavi P. Patwari' ${ }^{1}$, Tracey M. Stewart ${ }^{1}$, Casey M. Rand ${ }^{1}$, Michael S. Carroll ${ }^{1}$, Nancy L. Kuntz ${ }^{2}$, Anna S. Kenny ${ }^{1}$, Cindy D. Brogadir ${ }^{1}$ \\ and Debra E. Weese-Mayer ${ }^{1}$
}

INTRODUCTION: Congenital central hypoventilation syndrome (CCHS) is characterized by alveolar hypoventilation, autonomic nervous system (ANS) dysregulation (ANSD), and mutations in the paired-like homeobox 2B (PHOX2B) gene. ANSD in CCHS affects multiple systems and includes ophthalmologic abnormalities. We hypothesized that quantitative pupil measures, obtained using pupillometry, would vary between cases with CCHS and controls and within those with CCHS by PHOX2B genotype.

RESULTS: Measures known to be illustrative of sympathetic and parasympathetic response (prestimulus, maximum pupil diameter, percentage of pupil constriction after light stimulus, and average constriction and dilation velocities) were significantly reduced in those with CCHS as compared with controls (all $P<0.05$ ).

DISCUSSION: These reductions are indicative of both sympathetic and parasympathetic deficits in CCHS, which is in keeping with the role of $P H O X 2 B$ in ANS development. An inverse linear relationship was apparent in pupil diameter and velocity measurements among the cases with CCHS with the most common heterozygous $P H O X 2 B$ polyalanine expansion repeat mutations, suggesting a graded phenotype/genotype dose response based on polyalanine repeat length. These results confirm our central hypotheses while offering the first objective measures of pupillary dysfunction and ophthalmologicspecific ANSD in CCHS.

METHODS: A total of 316 monocular measurements were taken under dark-adapted conditions with a fixed light stimulus from 22 PHOX2B mutation-confirmed cases with CCHS and 68 healthy controls.

ongenital central hypoventilation syndrome (CCHS) is characterized by central alveolar hypoventilation with autonomic nervous system dysregulation (ANSD) and is diagnosed in the absence of primary pulmonary, cardiac, or neuromuscular disease or a brainstem lesion that can account for the full phenotype (1). Discovery of the CCHS disease-defining gene, pairedlike homeobox $2 \mathrm{~B}$ (PHOX2B) (2-4), and appreciation of its role in both embryologic development and regulatory function of the autonomic nervous system (ANS) (5-7), has broadened the understanding of the CCHS phenotype. The majority of individuals with CCHS are heterozygous for $\mathrm{PHOX} 2 \mathrm{~B}$ polyalanine repeat expansion mutations (PARMs), with a range of 24-33 alanine bases on the affected allele (20 repeats is normal; normal genotype is 20/20). Missense, nonsense, frameshift, and stop codon mutations in the $P H O X 2 B$ gene, referred to as nonpolyalanine repeat expansion mutations (NPARMs), account for the remaining mutations in CCHS. Quantitative assessment of the respiratory control and ANSD features of CCHS by $\mathrm{PHOX} 2 \mathrm{~B}$ genotype has identified a graded genotype-phenotype relationship between mutation repeat length and ventilatory requirements $(3,4)$, Hirschsprung disease $(8,9)$, neural crest tumors $(8,9)$, and cardiac sinus pauses $(10)$.

Detailed understanding of the ANSD features in CCHS requires targeted investigation with objective measures of all organ systems served by the ANS. Ophthalmologic abnormalities that have been described among children with CCHS include miotic pupils, "poor" pupil reaction to light (not quantified), strabismus, convergence insufficiency, and, less commonly, larger pupils, anisocoria, and irregular-shaped pupils (11-13). However, no quantitative evaluation of pupillary response has been reported among children with $P H O X 2 B$ mutation-confirmed CCHS. Pupillometry offers noninvasive, rapid, and objective measurements of pupillary function; these measurements serve as an ideal instrument to quantify ophthalmologic-specific ANSD. Pupillometry has been successfully used to evaluate ANSD in autonomic neuropathies (14-16), pediatric enuresis (17), heart failure (18), and familial dysautonomia (19) and has the potential for use in other pediatric disorders of respiratory and autonomic dysregulation. Although multiple factors can influence pupil diameter and response, with standardized conditions and intact afferent pathways, pupillometry allows for insight into efferent ANS pathways (14). Specifically, these include the sympathetic nervous system (SNS) component via pupillary dilation response and the parasympathetic nervous system (PSNS) component via pupillary constriction (Figure 1).

\footnotetext{
'Department of Pediatrics, Center for Autonomic Medicine in Pediatrics, Children's Memorial Hospital, Northwestern University Feinberg School of Medicine, Chicago, Illinois; ${ }^{2}$ Department of Neurology, Children's Memorial Hospital, Northwestern University Feinberg School of Medicine, Chicago, Illinois. Correspondence: Pallavi P. Patwari (PPatwari@ChildrensMemorial.org) 


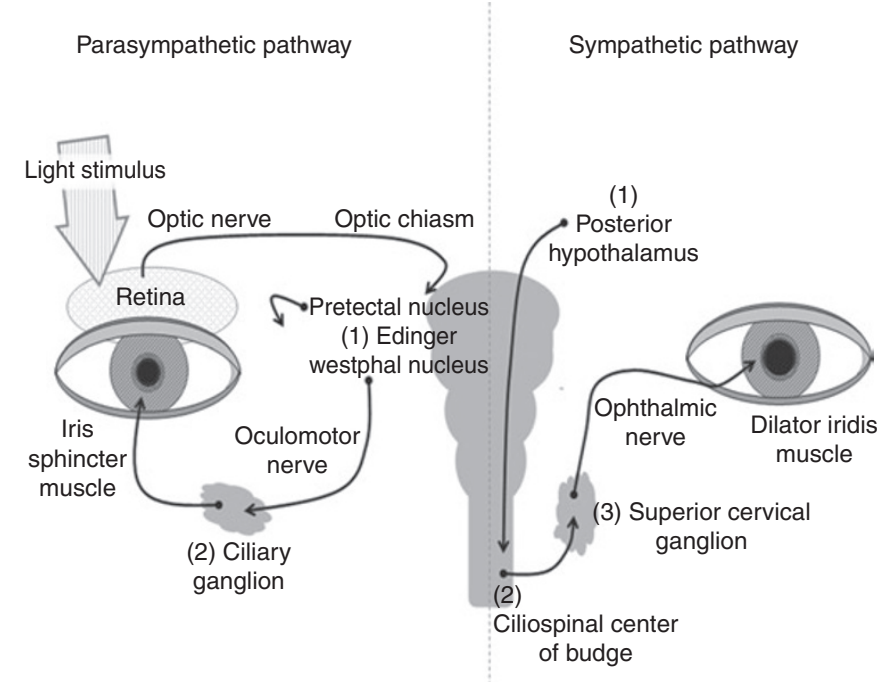

Figure 1. Schematic of the sympathetic nervous system (SNS) and the parasympathetic nervous system (PSNS) pathways. The SNS pathway begins in the hypothalamus, descends through the brain stem and cervical spinal cord to synapse in the ciliospinal center of Budge; it then exits the nerve root and travels to the superior cervical ganglion (preganglionic). The postganglionic pathway travels via the ophthalmic nerve to the pupil dilator muscle, resulting in pupil dilation. For the PSNS, the afferent pathway initiates after a light stimulus and travels from the retina to the optic chiasm to the pretectal nucleus in the midbrain. The PSNS efferent pathway begins in the Edinger-Westphal nucleus in the dorsal midbrain. The preganglionic neuron travels via the oculomotor nerve (cranial nerve III) to the ciliary ganglion. Then, the postganglionic signal travels to the iris sphincter muscle, resulting in pupil constriction.

We hypothesized that objective pupillary measures with pupillometry in cases with CCHS would vary as compared with controls and among those with CCHS by $\mathrm{PHOX} 2 \mathrm{~B}$ genotype, demonstrating deficits in both SNS and PSNS responses.

\section{RESULTS}

\section{Cases With CCHS}

A total of 189 pupillary measurements, 94 from the right eye and 95 from the left (including three single, unpaired measurements), were taken from 22 PHOX $2 B$ mutation-confirmed cases (mean age $9.5 \mathrm{y}$; range $0.4-24.7 \mathrm{y}$ ). $\mathrm{PHOX} 2 \mathrm{~B}$ mutations among cases with CCHS include genotypes 20/25, 20/26, 20/27, and 20/33 for PARMs and an 8-bp duplication and a 38-bp deletion for NPARMs. One child (at $5.8 \mathrm{y}$ ) was unable to cooperate sufficiently while performing pupillometry measurements and another child was able to cooperate during infancy, but not at ages of 9 and $15 \mathrm{mo}$.

All the subjects met the diagnostic criteria for CCHS based on the 2010 American Thoracic Statement on CCHS (1) and together represent a broad spectrum of disease. Of the 22 cases, $45 \%(n=10)$ require ventilatory support while asleep and when awake, $55 \%(n=12)$ require night-only ventilatory support, $41 \%$ $(n=9)$ have Hirschsprung disease, and $41 \%(n=9)$ have a history of prolonged sinus pause requiring a cardiac pacemaker.

\section{Healthy Controls}

A total of 127 pupillary measurements, 65 from the right eye and 62 from the left (including nine single, unpaired measurements), were taken from 68 healthy controls (mean age 9.2 y; range $0.3-23.6$ y). Seven children (of $75=9.3 \%$ ) with an average age of $2.5 \mathrm{y}$ were unable to cooperate sufficiently to allow obtainment of pupillometry measurements.

\section{Record Review in Cases and Questionnaire Results in Controls}

Controls were excluded if there was known ophthalmologic or autonomic abnormality as determined from the screening ophthalmologic/ANSD questionnaire. Extensive medical record reviews were performed for all cases with CCHS; these reviews included any formal ophthalmologic reports and a detailed review of systems with a focus on ANSD. From these, the most common findings were associated strabismus, convergence insufficiency, and mild refractive abnormalities. Aside from these, no apparent ocular or anterior visual pathway defects were noted. Nine of $22(41 \%)$ cases with CCHS and 19 of 68 controls (28\%) wore prescribed corrective lenses or glasses. In consideration of refractive variance, we found no statistical difference in pupillary measurements between those children who had glasses and those who did not, with analysis of the total cohort (cases and controls combined).

\section{Overall Pupillometry Measures}

A total of 316 monocular, pupillary measurements were taken from cases with CCHS and controls. The characteristics of all cases, cases by $P H O X 2 B$ genotype, and controls are summarized in Table 1. Anisocoria with a difference of $\geq 1 \mathrm{~mm}$ between maximum right and maximum left pupil diameter by standard clinical definition was found in two cases with CCHS and one control. Even with more stringent criteria (difference $>0.4 \mathrm{~mm}$ at any measurement), anisocoria was found in $28 \%$ (33 of 117) of measurements in those with CCHS and 22\% (13 of 59) of measurements in controls, both within the $41 \%$ reported prevalence of anisocoria in healthy individuals (20). Measurements for both eyes were combined and used for further analysis in both cases and controls. Mean pupillary measurements from pupillometry in all cases with CCHS, cases by genotype, and controls are shown in Table 2.

The pupil measurements at dark-adapted baseline $(\max )$, in response to light ( $\mathrm{min}$, average constriction velocity (ACV), percentage of pupil constriction (delta)), and recovery from light exposure (average dilation velocity (ADV)) for cases with CCHS demonstrate smaller pupil diameters (max, min; Figure 2a), reduced velocity measures (ACV, ADV; Figure 2b), and reduced pupil constriction (delta; Figure 2c) as compared with controls (Table 2). The mean latency of constriction was longer in all cases with CCHS as compared with controls (Table 2). Within CCHS (Figure 3a-c), there is an inverse linear relationship for max, min, delta, ACV, and ADV among the most common PHOX2B genotypes (20/25, 20/26, and 20/27), such that each additional alanine results in greater impairment in pupillary measure and response.

\section{Pupillometry Measures Reflecting ANS Response}

Pupillometry measures of SNS response relate to pupil dilation and include max and ADV (Table 3), and measures of PSNS 


\section{Articles | Patwarietal.}

Table 1. Cohort characteristics: cases with CCHS and controls

\begin{tabular}{|c|c|c|c|c|c|c|c|}
\hline \multirow[b]{2}{*}{ Genotype } & \multirow{2}{*}{$\begin{array}{c}\text { Controls } \\
-\end{array}$} & \multirow{2}{*}{$\begin{array}{c}\text { All CCHS } \\
-\end{array}$} & \multicolumn{5}{|c|}{ CCHS by $P H O X 2 B$ genotype } \\
\hline & & & $20 / 25$ & $20 / 26$ & $20 / 27$ & $20 / 33$ & NPARM \\
\hline Measurements & 127 & 189 & 48 & 80 & 52 & 3 & 6 \\
\hline Gender (\% female) & 53 & 45 & 71 & 43 & 43 & 0 & 0 \\
\hline \multicolumn{8}{|l|}{ Ethnicity $^{\mathrm{a}}$} \\
\hline$\%$ Hispanic & 21 & 9 & 14 & 14 & - & - & - \\
\hline$\%$ Asian & 6 & 5 & 14 & - & - & - & - \\
\hline \multicolumn{8}{|l|}{ Age (years) } \\
\hline Median & 7.1 & 7.7 & 7.7 & 7.8 & 12.1 & 0.4 & 7.5 \\
\hline Range & $0.3-23.6$ & $0.4-24.7$ & $3.3-16.8$ & $4.6-17.6$ & $3.7-24.7$ & - & $3.4-11.6$ \\
\hline
\end{tabular}

CCHS, congenital central hypoventilation syndrome; NPARM, non-polyalanine repeat expansion mutations.

apost-hoc analysis revealed no statistical difference between Caucasian and Hispanic groups for any pupillary measures.

Table 2. Mean pupillometry measurements in cases with $\mathrm{CCHS}$ and healthy controls

\begin{tabular}{|c|c|c|c|c|c|c|c|}
\hline \multirow[b]{2}{*}{ Genotype } & \multirow{2}{*}{$\begin{array}{c}\text { Controls } \\
-\end{array}$} & \multirow{2}{*}{$\begin{array}{c}\text { All CCHS } \\
-\end{array}$} & \multicolumn{5}{|c|}{ CCHS by $P H O X 2 B$ genotype } \\
\hline & & & $20 / 25$ & $20 / 26$ & $20 / 27$ & $20 / 33$ & NPARM \\
\hline $\operatorname{Max}(\mathrm{mm})$ & $6.6 \pm 0.9$ & $5.0 \pm 1.3^{* *}$ & $6.4 \pm 0.6$ & $5.1 \pm 0.9$ & $3.5 \pm 0.6$ & 4.2 & $3.5 \pm 0.3$ \\
\hline Delta (\%) & $28 \pm 7$ & $16 \pm 10^{* *}$ & $25 \pm 4$ & $17 \pm 5$ & $9 \pm 8$ & 4 & $8 \pm 6$ \\
\hline $\mathrm{ACV}(\mathrm{mm} / \mathrm{s})$ & $3.51 \pm 0.6$ & $1.64 \pm 1.16^{* *}$ & $2.94 \pm 0.64$ & $1.75 \pm 0.46$ & $0.56 \pm 0.66$ & 0.25 & $0.28 \pm 0.24$ \\
\hline Latency (s) & $0.23 \pm 0.03$ & $0.26 \pm 0.08^{*}$ & $0.25 \pm 0.02$ & $0.29 \pm 0.02$ & $0.26 \pm 0.15$ & 0.19 & $0.24 \pm 0.07$ \\
\hline
\end{tabular}

Values for all cases with CCHS and controls include mean \pm SD. P values are based on case-control (all cases with CCHS vs. controls) comparisons using two-tailed $t$-tests assuming unequal variance.

ACV, average constriction velocity; ADV, average dilation velocity; CCHS, congenital central hypoventilation syndrome; NPARM, non-polyalanine repeat expansion mutations.

${ }^{*}$ P value $<0.05 ;{ }^{* *} P$ value $<0.005$

response relate to pupil constriction and include delta and ACV (Table 4). In comparison with controls, each CCHS genotype consistently demonstrated lower max, ADV, delta, and ACV, except for CCHS $20 / 25$. The time to reach $75 \%$ of starting diameter, or $\mathrm{T}_{3 / 4}$, reflects the late redilation response and indicates SNS drive. We found that the pupil did not redilate sufficiently to calculate this in 31 of $127(24 \%)$ measurements in controls and in 55 of 189 (29\%) measurements in those with CCHS. The frequency of this occurrence was variable among individuals with CCHS by genotype: 3 of 7 for the 20/25 genotype, 7 of 7 for 20/26, and 4 of 5 for the 20/27 genotype.

\section{DISCUSSION}

These findings are the first to describe pupil abnormalities in CCHS within the context of PHOX2B mutations, demonstrate a correlation between the PHOX2B genotype and CCHS pupillary response phenotype, and quantify the sympathetic and parasympathetic components of pupillary dysregulation among children with CCHS.

Published reports of pupillary abnormalities in CCHS include a description of miosis (as demonstrated in this study) as well as mydriasis $(11,21)$ (which we did not find in our cohort). This seeming inconsistency is likely due to the limitation in confirming CCHS diagnosis with genetic testing in previous studies as $P H O X 2 B$ clinical testing was made available only in 2003 (1). Although there is a possibility that the genetic variability among NPARMs may be associated with variable ophthalmologic phenotype, elucidation was limited due to the small NPARM sample size of this study. Finally, the previously reported pupil findings were made in the absence of objective measurements or controlled stimulus presentation, so specific comparison with our pupillometry results cannot be made. Published reports of pupillary abnormalities in children with familial dysautonomia, another disorder of respiratory and autonomic regulation, also demonstrate a decrease in both SNS and PSNS response, but direct comparison is limited due to variation in testing protocol (19).

Pupillometry measurements of SNS response related to pupil dilation include maximum pupil diameter and ADV, and measures of PSNS function related to pupil constriction include delta and ACV (Tables 3 and 4). With activation of the SNS, two signals are initiated. An inhibitory signal releases constriction of the iris sphincter; simultaneously, an excitatory signal from the hypothalamus travels down the cervical cord and out to the superior cervical ganglion and then to the ophthalmic nerve to signal contraction of the dilator muscle, 
a

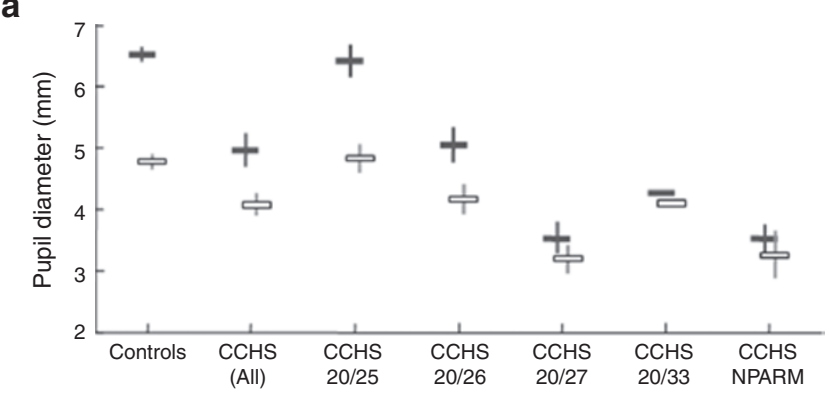

b

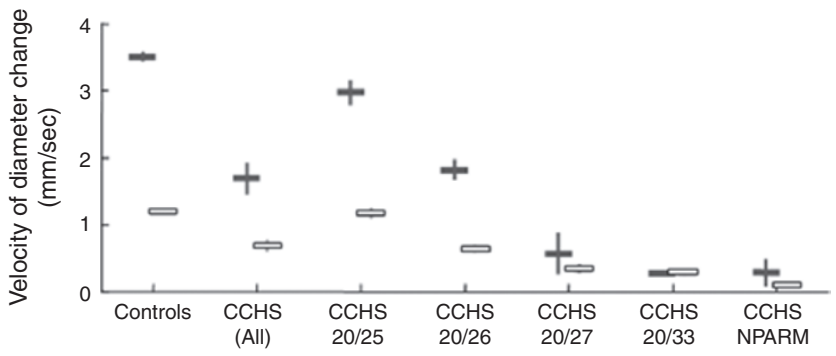

C

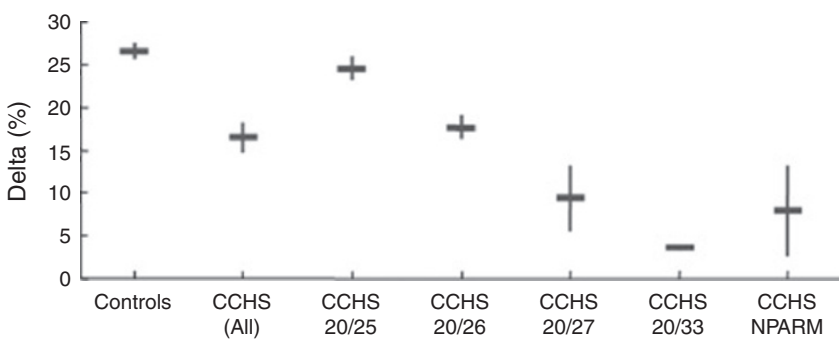

Figure 2. Pupillometry measurements in 22 subjects with CCHS and 68 healthy controls. (a) Maximum and minimum pupil diameter. The average maximum pupil diameter (dark horizontal bar) and average minimum pupil diameter (white horizontal bar) is shown for each group. The vertical bars represent standard error of the mean (SEM). (b) Average pupil constriction and dilation velocities. Average constriction velocity (dark horizontal bar) and average dilation velocity (white horizontal bar) for each group are shown. Vertical bars represent SEM. (c) Percentage of pupil constriction (delta). The average delta, or percentage difference between maximum and minimum pupil diameter, of each group is represented by the horizontal bar. Vertical bars represent SEM. CCHS, congenital central hypoventilation syndrome; NPARM, non-polyalanine repeat expansion mutations.

thereby causing pupil dilation. With light stimulus, an afferent signal from the retina travels to the midbrain (pretectal then Edinger-Westphal nuclei), thereby activating the PSNS. An efferent signal travels bilaterally via the oculomotor nerve and ciliary ganglion to signal contraction of the iris sphincter, thus causing pupil constriction (Figure 1).

The $\mathrm{T}_{3 / 4}$ measure indicates the late redilation response and more closely reflects the degree of sympathetic drive but is also strongly influenced by the amplitude of the light reflex (delta). For this study, we chose to use measures of max and ADV to represent SNS response because of the high frequency of absent $\mathrm{T}_{3 / 4}$ values from our cohort (cases and controls with insufficient redilation and with disproportionate representation in the CCHS 20/26 and 20/27 genotypes) and significant amount of intraindividual variability when a value was calculated. Therefore, the analysis of $\mathrm{T}_{3 / 4}$, with exclusion of many of the potentially longest $T_{3 / 4}$ values would inaccurately reflect the late dilation response. In theory, this is an important measure, but in practice $\mathrm{T}_{3 / 4}$ seems to be a difficult measure to obtain in our cohort of children.

The evidence for both sympathetic and parasympathetic dysregulation in CCHS follows logically from a basic understanding of the PHOX2B gene role in embryologic development of the sympathetic, parasympathetic, and enteric pathways of the ANS $(5,22)$. We postulate that the demonstrated functional differences in pupillary measures are specific to the ANS efferent pathways. This is based on the absence of afferent defects as observed from a review of the records and demonstration that refractive variance (as demonstrated by the need or a lack of it for glasses) was not associated with differences in pupil measurements. $P H O X 2 B$ expression patterns in mouse knockout models indicate a lack of $P H O X 2 B$ expression at the level of the midbrain (Edinger-Westphal nucleus, pretectal nucleus) and hypothalamus (22). Consequently, we propose that pupillometry measures obtained in genetically confirmed CCHS can be used to elucidate efferent ophthalmologic ANS dysfunction and may have applicability in the evaluation of efferent pathways of other autonomic disorders.

The limitations of pupillometry as a tool include the fact that the pupillary response is affected by multiple factors indicating potential deficits at each step of the pathway (Figure 1), which cannot be easily teased out. Specifically, ocular pathology and anterior visual pathway disease, which could potentially affect pupillary response, were not formally investigated in our study and related information is limited to what we could obtain from a review of the medical record. Considering this limitation, analysis was performed to determine if subtle visual pathway deficits (such as with refractive variance requiring corrective lenses) would affect pupillary measurements and we found no association. Furthermore, although we can extrapolate an understanding of the isolated roles of the SNS and PSNS, the pupillometry measurements truly represent a balance of the SNS and PSNS responses. In addition, despite being rapid and noninvasive, patient cooperation is necessary to obtain interpretable results from pupillometry. Therefore, our study is limited to only those children who were sufficiently cooperative to participate. Not surprisingly, the average age of those excluded was $2.5 \mathrm{y}$, suggesting that toddlers have the most difficulty with cooperating sufficiently to complete pupillometry. Finally, the resting or maximum pupil diameter can be influenced by behavioral factors, with larger pupils associated with pain, fear, excitement, and engagement. By providing a consistent testing environment for all cases and controls and by obtaining multiple measurements from each of our patient with CCHS, the variability due to these factors should be reduced as much as possible.

The practical implications of these results are threefold. First, among individuals with CCHS, pupillometry has potential utility in expanding our understanding of the phenotypic spectrum by accurately and objectively documenting the pupillary measurements in even the mildest cases. Second, and more broadly, the sensitivity of pupillometry in differentiating the CCHS phenotype shown in this study suggests 

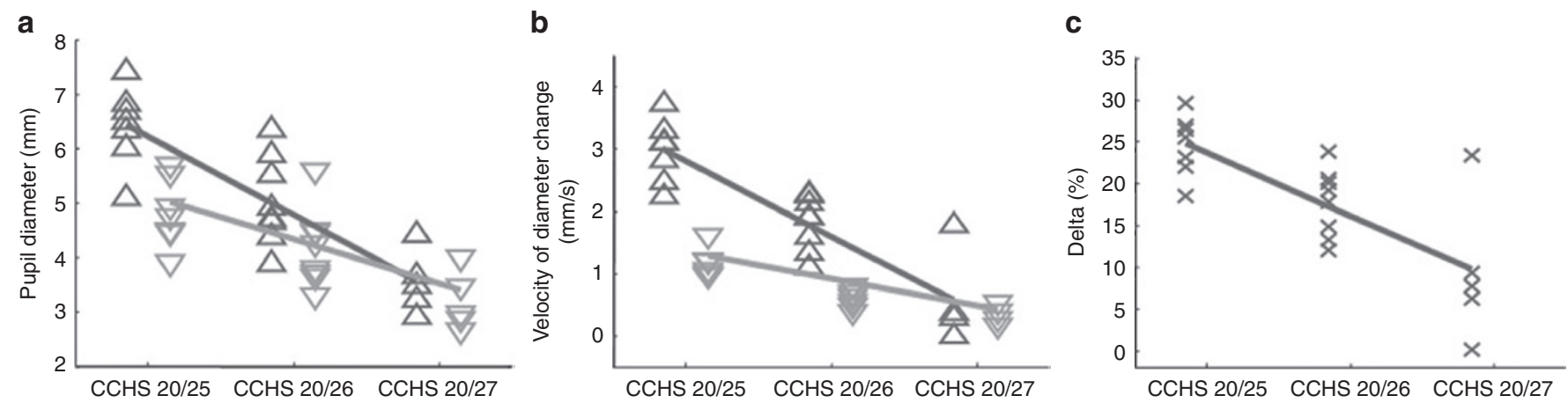

Figure 3. Pupillometry measurements among subjects with $C C H S$ by $P H O X 2 B$ genotype. $P H O X 2 B$ is the disease-defining gene for $C C H S$ that plays a key role in embryologic development of the autonomic nervous system. In normal individuals, there are 20 alanine repeats. In CCHS, the most common mutations are heterozygous polyalanine repeat expansion mutations (PARMs) causing expansion from 24 to 33 repeats on the affected allele. Among PARMs, the most common genotypes are 20/25, 20/26, and 20/27. (a) Maximum and minimum pupil diameter. The average maximum pupil diameter $(\Delta)$ and average minimum pupil diameter $(\nabla)$ for each case with CCHS are shown by PHOX2B genotype groups (genotypes 20/25, 20/26, and 20/27). (b) Average pupil constriction and dilation velocities. Average constriction velocity $(\Delta)$ and average dilation velocity $(\nabla)$ for each case with $C C H S$ are shown by PHOX2B genotype groups. (c) Percentage of pupil constriction (delta). The average delta $(\mathrm{X})$, or percentage difference between maximum and minimum pupil diameter, of each case with $\mathrm{CCHS}$ is shown by PHOX2B genotype groups. CCHS, congenital central hypoventilation syndrome.

Table 3. Measures of sympathetic nervous system response: comparison among cases with CCHS by $P H O X 2 B$ genotype and controls

\begin{tabular}{lcccccccc}
\hline & \multicolumn{3}{c}{$\begin{array}{c}\text { Maximum pupil } \\
\text { diameter (max) }\end{array}$} & & & \multicolumn{3}{c}{$\begin{array}{c}\text { Average dilation } \\
\text { velocity (ADV) }\end{array}$} \\
\cline { 2 - 4 } \cline { 6 - 8 } \cline { 5 - 7 } & $20 / 25$ & $20 / 26$ & $20 / 27$ & & $20 / 25$ & $20 / 26$ & $20 / 27$ \\
\hline Controls & NS & $*$ & $* *$ & & NS & $* *$ & $* *$ \\
$20 / 25$ & & $*$ & $* *$ & & $*$ & $* *$ \\
$20 / 26$ & & & & $*$ & & & $*$ \\
\hline
\end{tabular}

SNS response is reflected in measurements related to pupil dilation (max and ADV). ANOVA was completed by genotype with subsequent intergenotype $t$-test analysis, assuming unequal variance.

CCHS, congenital central hypoventilation syndrome; NS, not significant; SNS, sympathetic nervous system.

${ }^{*} P$ value $<0.05 ; * *$ value $<0.001$

Table 4. Measures of parasympathetic nervous system response: comparison among cases with CCHS by $P H O X 2 B$ genotype and controls

\begin{tabular}{lcccccccc}
\hline & \multicolumn{3}{c}{$\begin{array}{c}\text { Percent of pupil } \\
\text { constriction (delta) }\end{array}$} & & & \multicolumn{3}{c}{$\begin{array}{c}\text { Average constriction } \\
\text { velocity }\end{array}$} \\
\cline { 2 - 4 } \cline { 6 - 8 } \cline { 6 - 8 } & $20 / 25$ & $20 / 26$ & $20 / 27$ & & $20 / 25$ & $20 / 26$ & $20 / 27$ \\
\hline Controls & NS & $*$ & $*$ & & $*$ & $* *$ & $*$ \\
$20 / 25$ & & NS & $*$ & & $*$ & $* *$ \\
$20 / 26$ & & & NS & & & & $*$ \\
\hline
\end{tabular}

PSNS response is reflected in measurements related to pupil constriction (delta and ACV). ANOVA was completed by genotype with subsequent intergenotype $t$-test analysis, assuming unequal variance.

CCHS, congenital central hypoventilation syndrome; NS, not significant; PSNS, parasympathetic nervous system.

${ }^{*} P$ value $<0.05 ; * *$ value $<0.001$

that it can be used to supplement formal autonomic testing in children (as an adjunct, rather than isolated tool). This includes the potential for pupillometry to predict risk for other pathology (i.e., risk for cardiac sinus pauses) as a sign of

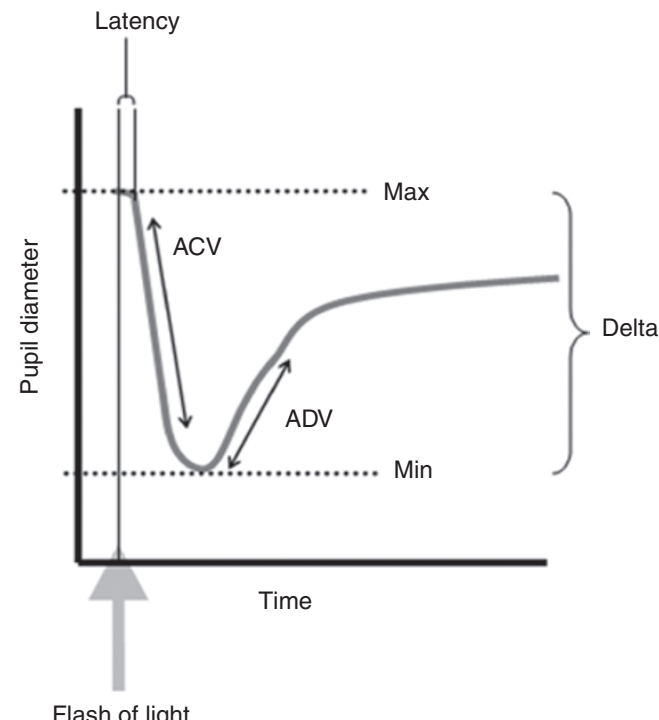

Figure 4. Pupillogram demonstrating pupillometry measurements in response to light. Pupillogram of pupil response to a single light stimulus with fixed intensity and with the following pupillary measurements is shown: ACV, average constriction velocity; $A D V$, average dilation velocity; delta, percentage of pupil constriction; max, maximum pupil diameter prior to light stimulus (dark-adapted conditions); min, minimum pupil diameter after light stimulus delivery.

cardiac ANSD (10). Accordingly, future steps would include systematic investigation of which, if any, phenotypic features in CCHS are associated with the various pupillary measurements. Third, pupillometry may be useful in providing quantitative measures in tracking general ANS response to intervention trials in CCHS and other disorders with autonomic dysregulation.

In conclusion, these results confirm our hypothesis that pupillometry measures will vary between those with CCHS and controls and within individuals with CCHS by $\mathrm{PHOX} 2 \mathrm{~B}$ genotype. Further inquiry into the specific mechanisms responsible 
for these findings may help elucidate the autonomic phenotype in other pediatric disorders within respiratory and autonomic dysregulation.

\section{METHODS}

\section{Cases With CCHS}

Diagnosis was confirmed in all cases with CCHS, with comprehensive clinical evaluation at the Center for Autonomic Medicine in Pediatrics at Children's Memorial Hospital. Pupillometry was performed daily as part of clinical care to objectively document pupillary size and response to light. Ophthalmologic reports and clinical records were reviewed to determine the presence or absence of afferent pupil defects. Presence of a PHOX2B mutation was confirmed in all cases with CCHS and genotype was determined through fragment or sequence analysis as described previously (4).

\section{Healthy Controls}

Healthy children without a history of ANSD were recruited and completed a brief ophthalmologic and ANSD questionnaire in addition to pupillometry. Exclusion criteria included the following: known pupil abnormality, medication that would affect pupil function, or insufficient cooperation to obtain reliable measurements.

\section{Institutional Review Board Approval}

The study was approved by the Children's Memorial Hospital Institutional Review Board. The approval included a review of clinically obtained data in subjects with CCHS and informed consent was obtained from controls.

\section{Pupillometry Testing Conditions and Measurements}

The NeurOptics PLR-2000 (NeurOptics, Irvine, CA) was used in all cases with CCHS and controls to obtain measurements. Testing was performed during the daytime when the child was fully awake and initiated after a minimum of 1-min adaptation to a completely dark and quiet room. The pupil response to a fixed amount $(180 \mu \mathrm{W})$ and duration $(33 \mathrm{~ms})$ of light was recorded for a 5 -s period in each eye separately. The NeurOptics PLR-2000 pupillometer has an accuracy of $\pm 0.1 \mathrm{~mm}$, with measurements taken every $0.033 \mathrm{~s}$. Each monocular measurement included the following: maximum dark-adapted pupil diameter (max), minimum pupil diameter ( $\mathrm{min}$ ) after light exposure, delta, ACV, and ADV, and latency to pupil constriction (Figure 4). Multiple daily measurements in an individual with CCHS were averaged and that single set of values was used for further analysis, whereas one-time measurements from each eye were used for analysis in the healthy controls. For both cases with CCHS and controls, measurements from the right and left eyes were grouped together.

\section{Statistical Analyses}

Measurements from the cases with CCHS and controls were compared using unpaired, two-tailed $t$-tests assuming unequal variances. ANOVA was used for comparisons among cases with the most common PHOX2B genotypes $(20 / 25,20 / 26,20 / 27)$ with subsequent $t$-test analysis. Because of the small sample size, other PHOX2B mutations (20/33, NPARM: 8-bp duplication, and NPARM: 38-bp deletion) were excluded from further analysis. A probability value $<0.05$ was considered statistically significant.

\section{ACKNOWLEDGMENTS}

We gratefully acknowledge the children and their families who participated in this study. The authors also acknowledge and extend gratitude to Mark Wainright (head of Pediatric NeuroCritical Care at Children's Memorial Hospital) and Nicholas Volpe (head of Neuro-Ophthalmology at Northwestern University) for their encouragement, guidance, and valuable comments that have allowed completion of this study.

\section{STATEMENT OF FINANCIAL SUPPORT}

A portion of C.M.R. salary is supported by the Chicago Community Trust Foundation PHOX2B Patent Fund.

\section{REFERENCES}

1. Weese-Mayer DE, Berry-Kravis EM, Ceccherini I, Keens TG, Loghmanee DA, Trang H. An official ATS clinical policy statement: Congenital central hypoventilation syndrome: genetic basis, diagnosis, and management. Am J Respir Crit Care Med 2010;181:626-44.

2. Amiel J, Laudier B, Attié-Bitach T, et al. Polyalanine expansion and frameshift mutations of the paired-like homeobox gene PHOX2B in congenital central hypoventilation syndrome. Nat Genet 2003;33:459-61.

3. Matera I, Bachetti T, Puppo F, et al. PHOX2B mutations and polyalanine expansions correlate with the severity of the respiratory phenotype and associated symptoms in both congenital and late onset Central Hypoventilation syndrome. J Med Genet 2004;41:373-80.

4. Weese-Mayer DE, Berry-Kravis EM, Zhou L, et al. Idiopathic congenital central hypoventilation syndrome: analysis of genes pertinent to early autonomic nervous system embryologic development and identification of mutations in PHOX2B. Am J Med Genet A 2003;123A:267-78.

5. Howard MJ. Mechanisms and perspectives on differentiation of autonomic neurons. Dev Biol 2005;277:271-86.

6. Pattyn A, Morin X, Cremer H, Goridis C, Brunet JF. Expression and interactions of the two closely related homeobox genes Phox $2 \mathrm{a}$ and Phox $2 \mathrm{~b}$ during neurogenesis. Development 1997;124:4065-75.

7. Pattyn A, Morin X, Cremer H, Goridis C, Brunet JF. The homeobox gene Phox $2 b$ is essential for the development of autonomic neural crest derivatives. Nature 1999;399:366-70.

8. Berry-Kravis EM, Zhou L, Rand CM, Weese-Mayer DE. Congenital central hypoventilation syndrome: $P H O X 2 B$ mutations and phenotype. Am J Respir Crit Care Med 2006;174:1139-44.

9. Trochet D, O’Brien LM, Gozal D, et al. PHOX2B genotype allows for prediction of tumor risk in congenital central hypoventilation syndrome. Am J Hum Genet 2005;76:421-6.

10. Gronli JO, Santucci BA, Leurgans SE, Berry-Kravis EM, Weese-Mayer DE. Congenital central hypoventilation syndrome: $P H O X 2 B$ genotype determines risk for sudden death. Pediatr Pulmonol 2008;43:77-86.

11. Goldberg DS, Ludwig IH. Congenital central hypoventilation syndrome: ocular findings in 37 children. J Pediatr Ophthalmol Strabismus 1996;33:175-80.

12. Weese-Mayer DE, Shannon DC, Keens TG, Silvestri JM. Idiopathic congenital central hypoventilation syndrome: diagnosis and management. American Thoracic Society. Am J Respir Crit Care Med 1999;160:368-73.

13. Weese-Mayer DE, Silvestri JM, Menzies LJ, Morrow-Kenny AS, Hunt CE, Hauptman SA. Congenital central hypoventilation syndrome: diagnosis, management, and long-term outcome in thirty-two children. J Pediatr 1992;120:381-7.

14. Bremner F. Pupil evaluation as a test for autonomic disorders. Clin Auton Res 2009;19:88-101.

15. Bremner F, Smith S. Pupil findings in a consecutive series of 150 patients with generalised autonomic neuropathy. J Neurol Neurosurg Psychiatr 2006;77:1163-8.

16. Bremner FD, Smith SE. Pupil abnormalities in selected autonomic neuropathies. J Neuroophthalmol 2006;26:209-19.

17. Dundaroz R, Turkbay T, Erdem U, Congologlu A, Sakallioglu O, Tascilar E. Pupillometric assessment of autonomic nervous system in children with functional enuresis. Int Urol Nephrol 2009;41:231-5.

18. Keivanidou A, Fotiou D, Arnaoutoglou C, Arnaoutoglou M, Fotiou F, Karlovasitou A. Evaluation of autonomic imbalance in patients with heart failure: a preliminary study of pupillomotor function. Cardiol J 2010;17:65-72.

19. Dütsch M, Hilz MJ, Rauhut U, Solomon J, Neundörfer B, Axelrod FB. Sympathetic and parasympathetic pupillary dysfunction in familial dysautonomia. J Neurol Sci 2002;195:77-83.

20. Lam BL, Thompson HS, Corbett JJ. The prevalence of simple anisocoria. Am J Ophthalmol 1987;104:69-73.

21. Croaker GD, Shi E, Simpson E, Cartmill T, Cass DT. Congenital central hypoventilation syndrome and Hirschsprung's disease. Arch Dis Child 1998;78:316-22.

22. Patwari PP, Carroll MS, Rand CM, Kumar R, Harper R, Weese-Mayer DE. Congenital central hypoventilation syndrome and the $P H O X 2 B$ gene: a model of respiratory and autonomic dysregulation. Respir Physiol Neurobiol 2010;173:322-35. 